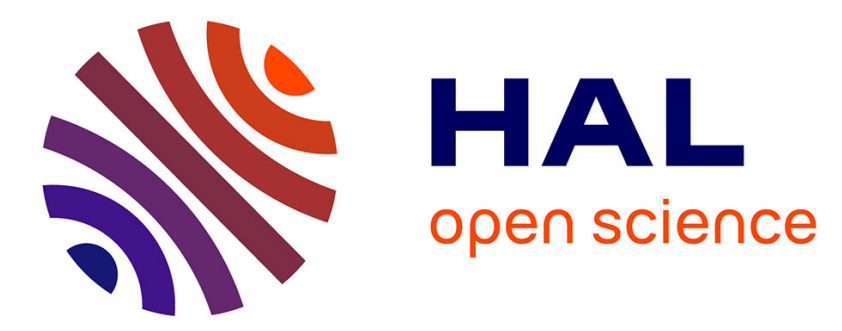

\title{
The motion of solid spherical particles falling in a cellular flow field at low Stokes number
}

Laurence Bergougnoux, Gilles Bouchet, Diego Lopez, Elisabeth Guazzelli

\section{To cite this version:}

Laurence Bergougnoux, Gilles Bouchet, Diego Lopez, Elisabeth Guazzelli. The motion of solid spherical particles falling in a cellular flow field at low Stokes number. Physics of Fluids, 2014, 26 (9), pp.093302. 10.1063/1.4895736 . hal-01066982

\section{HAL Id: hal-01066982 \\ https://hal.science/hal-01066982}

Submitted on 22 Sep 2014

HAL is a multi-disciplinary open access archive for the deposit and dissemination of scientific research documents, whether they are published or not. The documents may come from teaching and research institutions in France or abroad, or from public or private research centers.
L'archive ouverte pluridisciplinaire HAL, est destinée au dépôt et à la diffusion de documents scientifiques de niveau recherche, publiés ou non, émanant des établissements d'enseignement et de recherche français ou étrangers, des laboratoires publics ou privés. 


\title{
The motion of solid spherical particles falling in a cellular flow field at low Stokes number
}

\author{
Laurence Bergougnoux, Gilles Bouchet, ${ }^{a)}$ Diego Lopez, \\ and Élisabeth Guazzelli \\ Aix Marseille Université, CNRS, IUSTI UMR 7343, 13453 Marseille, France
}

(Received 10 April 2014; accepted 8 August 2014; published online 22 September 2014)

\begin{abstract}
We present a jointed experimental and numerical study examining the influence of vortical structures on the settling of solid spherical particles under the action of gravity at low Stokes numbers. The two-dimensional model experiment uses electroconvection to generate a two-dimensional array of controlled vortices which mimics a simplified vortical flow. Particle image-velocimetry and tracking are used to examine the motion of the particles within this vortical flow. Particle motion is compared to the predictions of a numerical simulation inspired by the model equation developed by Maxey ["The motion of small spherical particles in a cellular flow field," Phys. Fluids 30, 1915 (1987)]. @ 2014 AIP Publishing LLC. [http://dx.doi.org/10.1063/1.4895736]
\end{abstract}

\section{INTRODUCTION}

The transport of particles in a turbulent environment is relevant to many industrial and natural processes. Very often, the sedimentation of particles is a dominant phenomenon which is important to understand in a fundamental way. Examples include fluidized-bed reactors, the treatment of waste materials in clarifiers, the transport of sediment in rivers and estuaries, pyroclastic flows from volcanic eruptions, and bioconvection of plankton.

It is precisely in this latter context of plankton suspension in Langmuir circulation that Stommel ${ }^{1}$ made one of the first theoretical treatments of the settling of small particles in a cellular flow field. The cellular flow studied consisted of two-dimensional Taylor-Green vortices of size $L$ given by the stream function

$$
\psi=\frac{U_{0}}{k} \sin \left(k x_{1}\right) \sin \left(k x_{2}\right),
$$

where the wavenumber is given by $k=2 \pi / 2 L$. This simple flow was chosen as an idealized view of the wind-induced Langmuir cells occurring at the surface of lakes and oceans but could also be considered as mimicking a very simplified turbulent flow. Stommel ${ }^{1}$ neglected inertia in his study. The instantaneous particle velocity was then simply the sum of the local fluid velocity inferred from the stream function (1) and the Stokes settling velocity $V_{S}$. Stommel ${ }^{1}$ discussed the particle trajectories in terms of the ratio $W=V_{S} / U_{0}$ of the settling velocity $V_{S}$ to the vortex velocity $U_{0}$. For $W=0$ the particles simply move with the local fluid velocity as Lagrangian tracers. By contrast, for $W \geqslant 1$, settling dominates. For intermediate values of $W$, local fluid upward flow can be strong enough to induce particle suspension.

Maxey $^{2}$ extended the analysis to the inertial regime using the equation of motion for a small rigid sphere in a non-uniform flow field under general conditions. ${ }^{3,4}$ He performed a comprehensive study in terms of two additional dimensionless parameters: the ratio of the response time of the particle to the characteristic time $1 / k U_{0}$ of the flow field, i.e., the Stokes number $S t$, and a particle to fluid density parameter $R=\rho_{f} /\left(\rho_{p}+\rho_{f} / 2\right)$ where $\rho_{f}$ is the density of the fluid and $\rho_{p}$ that of the particles. The analysis was mostly focused on aerosol particles which are much denser than

a)Electronic mail: Gilles.Bouchet@univ-amu.fr 
the surrounding fluid $(R=0)$ and on light gas bubbles $(R=2)$. A linear stability analysis of the equilibrium points for the particles was also presented. In general, the equilibrium positions on the cell boundaries were seen to be unstable. As the density parameter $R$ approached the bubble limit of $R=2$, stable points were found in the interior of each vortical cell. The transition between instability and possible stability generally occurs when $R=2 / 3$ for which the particle is neutrally buoyant.

The above discussion leads us to introduce more formally the various dimensionless parameters involved in the problem. The fluid is characterized by its viscosity $\mu$ and density $\rho_{f}$, the particles by their radius $a$ and density $\rho_{p}$, the vortices by their wavenumber $k$ and velocity $U_{0}$, and finally the gravity force by the gravitational acceleration $g$. Dimensional analysis indicates that at least four dimensionless parameters have to be specified. An appropriate choice which follows that of Maxey ${ }^{2}$ is to consider the velocity ratio $W=V_{S} / U_{0}$ where the Stokes settling velocity is $V_{S}=\frac{2}{9}\left(\rho_{p}-\right.$ $\left.\rho_{f}\right) a^{2} g / \mu$, the density ratio is $R=\rho_{f} /\left(\rho_{p}+\rho_{f} / 2\right)$, the Stokes number is $S t=\frac{2}{9}\left(\frac{a^{2}}{\mu / \rho_{f}} k U_{0}\right) / R=$ $\frac{2}{9}\left(\rho_{p}+\rho_{f} / 2\right) a^{2} k U_{0} / \mu$ which is the ratio of the characteristic acceleration time of the particle and the convective time $1 / k U_{0}$, and the size ratio $P=k a$ which is often considered small. Other related dimensionless numbers can be of interest such as the Reynolds number of the settling particles $R e_{a}=$ $V_{S} a /\left(\mu / \rho_{f}\right)=\frac{9}{2} S t W R / P$ and that of the flow $R e_{k}=U_{0} /\left(k \mu / \rho_{f}\right)=R e_{a} /(P W)=\frac{9}{2} S t R / P^{2}$.

The cellular flow considered by Stommel ${ }^{1}$ and $\mathrm{Maxey}^{2}$ is in fact a simple but robust analytical framework for the study of turbulence, capturing key features of vortical effects on particles. An example is that of preferential sweeping, where particles heavier than the fluid settle along preferential paths in the inertial regime. ${ }^{5,6}$ This feature was observed by Wang and Maxey ${ }^{7}$ in fully turbulent flows, who also noted that preferential concentration was similar to that observed in cellular flows. ${ }^{8}$ Particle advection was studied at length in homogeneous turbulence both numerically ${ }^{9-13}$ and experimentally, ${ }^{14-16}$ showing in particular a significant increase of the average settling velocity of particles. While there has been a notable amount of numerical studies considering the model vortical flow proposed by Maxey, $2,17,18$ there is a lack of real experiments where single particles could be tracked while settling in the cellular flow and their trajectories compared individually to theoretical predictions. This is precisely the motivation for the present work.

In this paper, an experimental examination of the problem of the settling of spherical particles in a cellular flow field at low $S t$ is proposed. The experiment uses electroconvection to generate a twodimensional array of controlled vortices which resembles the cellular flow represented by the stream function (1). Comparison of the observed particle trajectories with the predictions of numerical simulations inspired by the model of Maxey ${ }^{2}$ provides (i) an examination of the different regimes of motion as well as (ii) a precise testing of the widely used equation of motion ${ }^{3,4}$ for a particle in a non-uniform flow at low Reynolds numbers called the Boussinesq-Basset-Oseen equation.

\section{EXPERIMENTS}

The experimental setup is shown in Figure 1. It consists of a tank made of Plexiglas ${ }^{\circledR}$ (of $50 \mathrm{~cm}$ height, $38 \mathrm{~cm}$ width, and $4 \mathrm{~cm}$ depth) filled with an aqueous mixture of citric acid, see Table I describing the characteristics of the different mixtures which have been used. The vortical flow is activated by electromagnetic forcing, i.e., an array of Laplace forces $\mathbf{j} \times \mathbf{B}$ where $\mathbf{j}$ is the electrical current density and $\mathbf{B}$ is the magnetic field. The magnetic field is produced by a checkerboard of permanent square magnets $\left(\mathrm{NdFeB}, B r \approx 1.3 \mathrm{~T}, 2 \times 2 \mathrm{~cm}^{2}\right)$ placed against the back wall of the tank of $2 \mathrm{~mm}$ thickness. The electrical current is driven between two carbon electrodes placed on opposite sides of the tank. Since the electrical current density $j$ is uniform, the spatial distribution of the electromagnetic forcing is determined by the position and size of the magnets. The blowup in Figure 1 gives a sketch of the mechanism for producing the network of Laplace forces (light gray arrows - red - along $x_{2}$ ): since the polarity (black arrows along $x_{3}$ ) of the permanent magnets alternates within the lattice square pattern, while the electrical current density is maintained constant (dashed arrows - green - along $x_{1}$ ), the Laplace forces are alternately oriented upward or downward. These Laplace forces give rise to a network of vortices, whose size is controlled by the size of the square magnets and whose intensity is controlled by the magnetic field of the permanent magnets, the intensity of the electric current, and the viscosity of the fluid. The maxima of velocity are located 

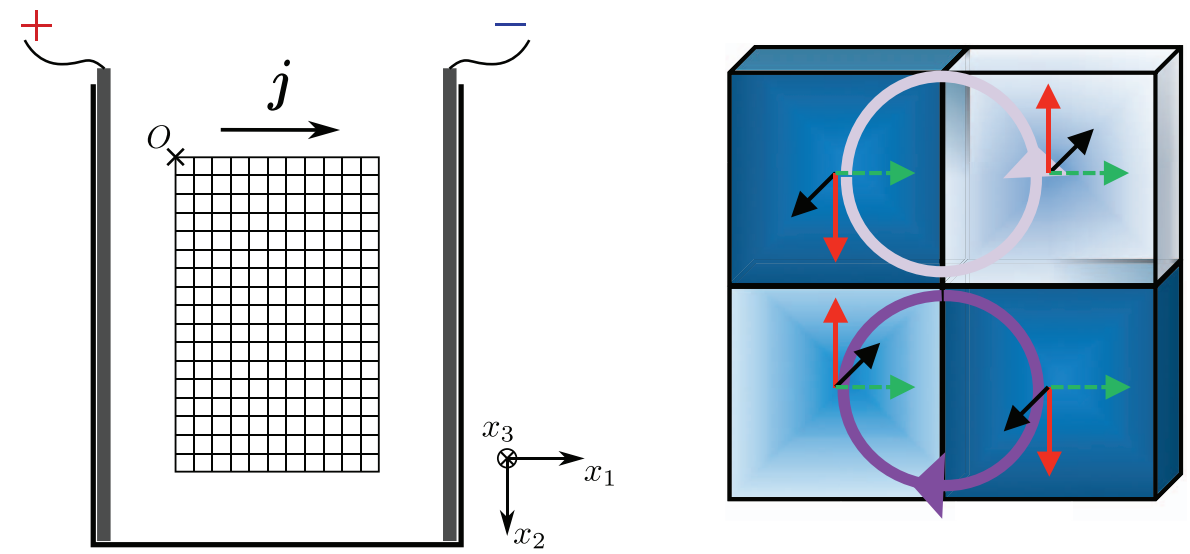

FIG. 1. Experimental setup and sketch of the network of Laplace forces (blowup). Magnets are placed against the back wall of the tank. The electrical current is driven between two carbon electrodes placed on opposite sides of the tank.

at the center of the magnets, while the minima are located on the edges. Electromagnetic forcing has been widely used to generate turbulent or chaotic two-dimensional flows ${ }^{19,20}$ or low-Reynoldsnumber cellular flows. ${ }^{21}$

The flow characterization is performed by particle image velocimetry (PIV) using the Matlab ${ }^{\text {TM }}$ PIV software DPIV soft. ${ }^{22}$ The fluid is seeded with hollow particles used as a fluid tracers (Dantec Measurement Technology, with diameter $\approx 15 \mu \mathrm{m}$ and density $\approx 1.4 \mathrm{~g} \mathrm{~cm}^{-3}$ ). The tank is illuminated by a green laser sheet (Laser $2000,532 \mathrm{~nm}, 100 \mathrm{~mW}$ ) aligned with the tank height. A digital camera (Pike F210B Allied Vision Technologies, $1920 \times 1080$ pixels $^{2}$ ) placed at right angle to the light-sheet in front of the tank is focused on the illuminated particles which scatter the light. Two images separated in time by typically $1 / 15-4 / 15 \mathrm{~s}$ are then recorded and processed using cross-correlations to find the velocity-vector map of the flow field. The spatial resolution of the measurement is given by the correlation window size $(\approx 1 / 5$ of the vortex size), while the flow-velocity resolution is given by the time separation between the two images. A typical velocity field is shown in Figure 2(b) and exhibits an array of stationary counterrotating vortices of the same size. Note that each vortex is discretized by $20 \times 20$ overlapping correlation windows. This array of counterrotating vortices generates a cellular flow with an array of stagnation points. Close to the stagnation points the flow can be considered as elongational, while close to the center of the vortices it can be seen as purely rotational. This cellular flow thus resembles the Taylor-Green cellular flow represented by the stream function (1). At low Reynolds number, the cellular flow produced by electroconvection is very close to a Taylor-Green cellular flow as can be seen in Figures 2(a) and 2(b). Discrepancies are seen with increasing Reynolds number (Figure 2(c)), but the flow remains stationary for $R e_{k} \lesssim 15$, i.e., in the range of Reynolds number considered in the experiments.

The generated flow is essentially two dimensional in the $\left(x_{1}, x_{2}\right)$ plane. However, a secondary three-dimensional flow may exist when the Reynolds number is significantly larger than one. ${ }^{23,24}$ This can be observed in Figure 3, showing PIV measurements in the $\left(x_{1}, x_{3}\right)$ plane at a horizontal edge of the magnets, for one of the largest Reynolds number examined $\left(R e_{k} \approx 7\right)$. The normal (horizontal) velocity $u_{3}$ is much lower than that of the vortices, and its maxima are located further from the magnet array. The intensity of the vortices reaches a maximum at about $5 \mathrm{~mm}$ from the tank wall,

TABLE I. Fluid characteristics: density $\rho_{f}$, viscosity $\mu$, electrical conductivity $\sigma$.

\begin{tabular}{lcccc}
\hline \hline Fluid & Mixture & $\rho_{f}\left(\mathrm{~g} \mathrm{~cm}^{-3}\right)$ & $\mu(\mathrm{P})$ & $\sigma\left(\mathrm{mS} \mathrm{cm}^{-1}\right)$ \\
\hline 1 & $60 \%$ water $+34 \%$ citric acid $+6 \%$ Ucon ${ }^{\mathrm{TM}}$ oil & 1.163 & 0.198 & 4.14 \\
2 & $64 \%$ water $+36 \%$ citric acid & 1.163 & 0.035 & 6.27 \\
3 & $61 \%$ water $+39 \%$ citric acid & 1.183 & 0.040 & 5.40 \\
\hline \hline
\end{tabular}




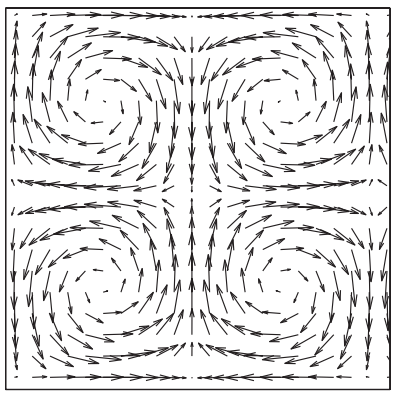

(a)

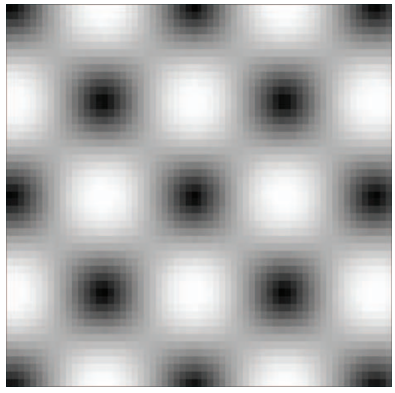

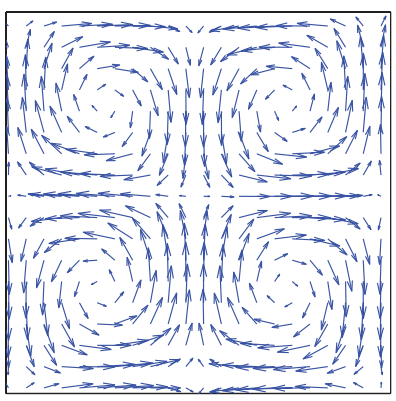

(b)

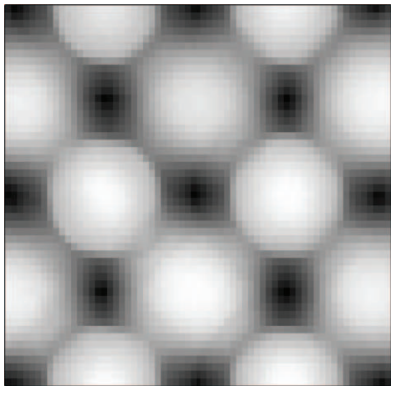

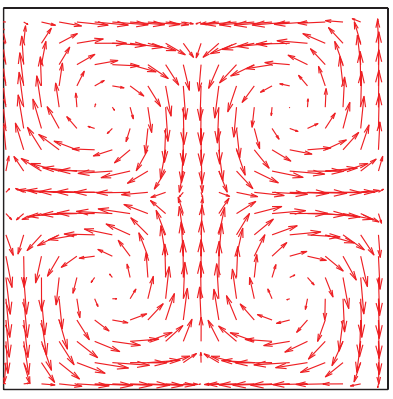

(c)

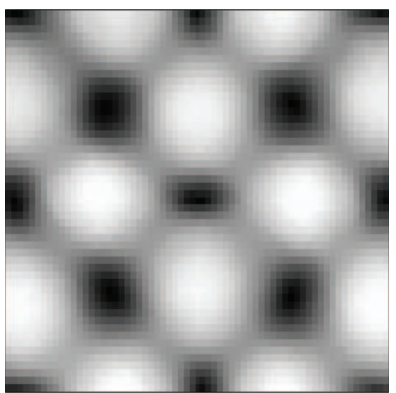

FIG. 2. Cellular flow generated by electro-convection versus Taylor-Green vortices. Velocity fields $\left(u_{1}, u_{2}\right)($ top line) and velocity norm (bottom line) for (a) Taylor-Green vortices, (b) experiments at $R e_{k} \approx 0.3$ (fluid 1) and (c) $R e_{k} \approx 8$ (fluid 2). Discrepancies with Taylor-Green model appear as the Reynolds number increases.

that is $7 \mathrm{~mm}$ from the magnets (Figure 3(b)), and at $x_{3}=-5 \mathrm{~mm}$, the normal (horizontal) velocity $u_{3}$ is negligible compared to the velocity of the vortices (Figure 3(c)). As a result, the trajectories of the sedimenting particles are examined in this vertical plane, where the flow can be considered as two-dimensional for the present range of Reynolds number $\left(0.3 \leq R e_{k} \leq 8\right)$. In addition, we have checked that the particle trajectories remained in this vertical plane by using a laser sheet as well as by observing that the sedimented particles at the bottom of the tank laid on a single straight line.

Particle tracking (PT) is used to examine the motion of the sedimenting particles within this vortical flow. Three different types of particles have been used in the experiments, see Table II. The particles are released at the top of the tank through a small vertical tube whose output can be positioned at different locations within the two-dimensional vortex array. Two side neon lights illuminate the front of the tank and the digital camera records the particle motions at 15 frame/s. The particle tracking scheme uses the circular Hough transform to detect the center of the particle. The experimental error in the particle center measurement is of $\approx 1 / 5$ of the particle diameter. Since the camera is kept at the same location in the PIV and PT measurements, the coordinates of the particle can be accurately positioned inside the velocity-vector map of the vortical flow as a function of time.
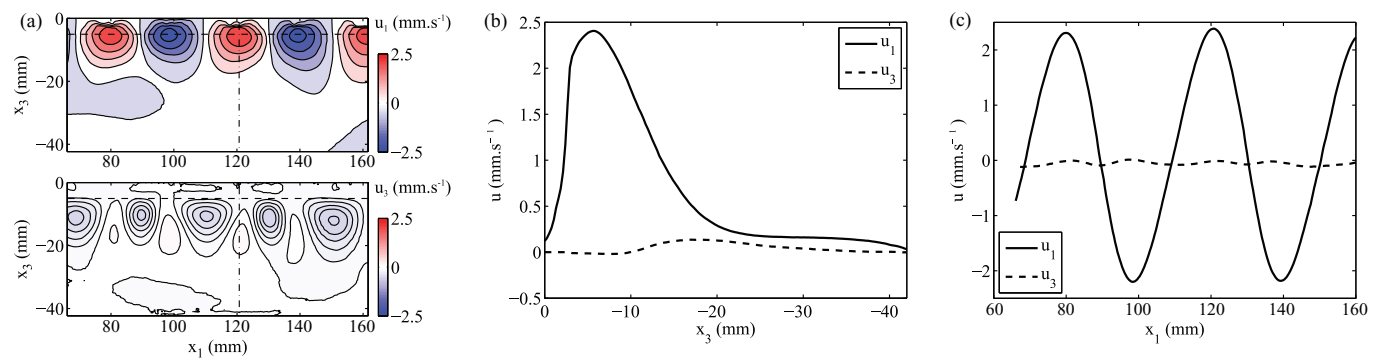

FIG. 3. (a) Velocities $u_{1}$ and $u_{3}$ measured by PIV in the $\left(x_{1}, x_{3}\right)$ plane between two rows of magnets. Velocity profiles (b) as a function of the distance to the magnets $\left[x_{1}=20 \mathrm{~mm}\right.$, dashed line in (a) $]$ and (c) along a line parallel to the magnets $\left[x_{3}=\right.$ $-5 \mathrm{~mm}$, dashed-dotted line in (a)]. Results obtained in fluid 2. 
TABLE II. Particle characteristics: mean sphere radius $a$ and density $\rho_{p}$.

\begin{tabular}{lcc}
\hline \hline Batch & $a(\mathrm{~cm})$ & $\rho_{p}\left(\mathrm{~g} \mathrm{~cm}^{-3}\right)$ \\
\hline$A$ & 0.0510 & 1.190 \\
$B$ & 0.1003 & 1.188 \\
$C$ & 0.3161 & 1.180 \\
\hline \hline
\end{tabular}

TABLE III. Dimensionless numbers for the different combinations of particles and fluids used in the experimental runs.

\begin{tabular}{lccccl}
\hline \hline Combination & $1 \mathrm{~A}$ & $1 \mathrm{~B}$ & $1 \mathrm{C}$ & $2 \mathrm{~B}$ & $3 \mathrm{~B}$ \\
\hline$R$ & 0.657 & 0.657 & 0.660 & 0.657 & 0.665 \\
$P$ & 0.080 & 0.158 & 0.497 & 0.158 & 0.158 \\
$S t$ & 0.0007 & 0.0027 & 0.0268 & 0.0673 & 0.0577 \\
$S t_{\mathrm{exp}}$ & 0.0006 & 0.0025 & 0.0239 & 0.0402 & 0.0412 \\
$S t_{\mathrm{SN}}$ & 0.0007 & 0.0025 & 0.0186 & 0.0434 & 0.0492 \\
$W$ & 0.878 & 3.205 & 21.646 & 4.133 & 0.668 \\
$W_{\exp }$ & 0.947 & 3.212 & 19.341 & 2.474 & 0.530 \\
$W_{\mathrm{SN}}$ & 0.862 & 3.005 & 14.973 & 2.635 & 0.567 \\
$R e_{a}$ & 0.023 & 0.163 & 3.472 & 5.221 & 0.732 \\
$R e_{k}$ & 0.329 & 0.323 & 0.323 & 8.017 & 6.962 \\
$\mathcal{L}$ & 60.191 & 62.654 & 62.938 & 3.256 & 3.404 \\
\hline \hline
\end{tabular}

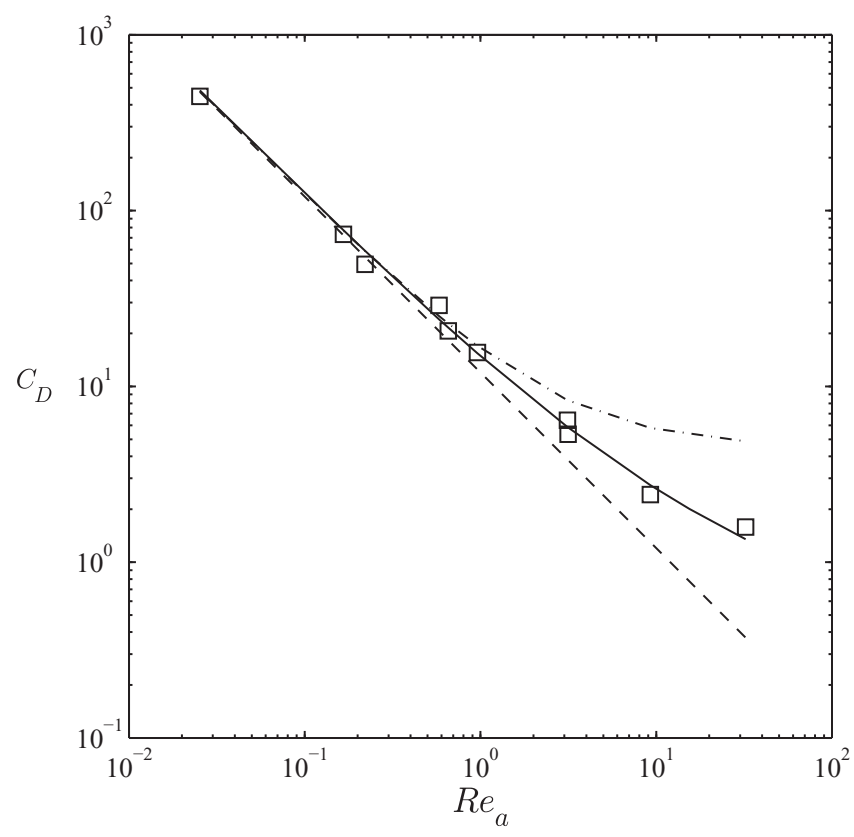

FIG. 4. Measured drag coefficient versus $R e_{a}(\square)$. The dashed line is the Stokes drag coefficient $12 / R e_{a}$, the dashed-dotted line is the Oseen drag coefficient $12\left(1+3 R e_{a} / 4\right) / R e_{a}$, and the solid line is the Schiller-Naumann drag coefficient $12[1+$ $\left.0.15\left(2 R e_{a}\right)^{0.687}\right] / R e_{a}$.

Particle trajectories are examined for different combinations of particles and fluid mixtures, see Table III which also shows the corresponding dimensionless parameters, $W, S t, R, P$. It is important to mention that the definitions of $W$ and $S t$ rely on assumption of a Stokes drag. In fact, as inertia is increased, the drag on settling particles deviates from the Stokes law. This has been inferred from measurements of particle settling velocities $V_{\exp }$ in the different fluids at rest and is shown in Figure 4. The Schiller-Naumann correlation agrees well with the experimental data. We have thus reported in Table III the modified $W_{\mathrm{SN}}$ and $S t_{\mathrm{SN}}$ which are built on the Schiller-Naumann drag as 
well as $W_{\text {exp }}$ and $S t_{\text {exp }}$. As expected from Figure 4, the Stokes numbers and dimensionless settling velocities agree well except for combination $1 \mathrm{C}$ for which the finite size of the particle may have some influence. Finally, we reported in Table III an additional dimensionless number noted $\mathcal{L}$, which is specific to the experimental setup, as it characterizes the role of the Laplace force used for flow generation, compared to particle acceleration by the flow, $\mathcal{L}=\frac{j B r}{\rho_{f} k U_{0}^{2}} R$.

\section{MODELING}

We consider the Boussinesq-Basset-Oseen equation including Faxen corrections ${ }^{3,4}$ for the motion of a spherical particle in a non-uniform or unsteady Stokes flow field $\mathbf{u}$,

$$
\begin{aligned}
m_{p} \frac{d \mathbf{v}}{d t} & =\left(m_{p}-m_{f}\right) \mathbf{g} \\
& +m_{f} \frac{D \mathbf{u}}{D t}-\frac{4}{3} \pi a^{3} \mathbf{j} \times \mathbf{B} \\
& -6 \pi \mu a\left(\mathbf{v}-\mathbf{u}-\frac{1}{6} a^{2} \nabla^{2} \mathbf{u}\right) \\
& -\frac{1}{2} m_{f} \frac{d \mathbf{v}}{d t}+\frac{1}{2} m_{f} \frac{D}{D t}\left(\mathbf{u}+\frac{1}{10} a^{2} \nabla^{2} \mathbf{u}\right) \\
& -6 \pi \mu a^{2} \int_{0}^{t} \frac{d\left(\mathbf{v}-\mathbf{u}-\frac{1}{6} a^{2} \nabla^{2} \mathbf{u}\right) / d \tau}{\sqrt{\pi v(t-\tau)}} d \tau,
\end{aligned}
$$

where $\mathbf{v}$ is the particle velocity and in addition to previously defined terms $m_{p}=\rho_{p} \frac{4}{3} \pi a^{3}$ the particle mass and $m_{f}=\rho_{f} \frac{4}{3} \pi a^{3}$ the mass of the displaced fluid. In this Eq. (2), $d / d t$ denotes the time derivative in a frame moving with the particle, while $D / D t$ the derivative following the fluid element. The forces included in the right-hand side of Eq. (2) are the buoyancy force, the force exerted by the undisturbed flow on the particle (fluid acceleration and Laplace force), the Stokes drag, the added mass effect, and the history Boussinesq-Basset force. The terms in $a^{2} \nabla^{2} \mathbf{u}$ are the Faxen corrections. Equation (2) is similar to that written by Maxey ${ }^{2}$ except for a correction in the added mass term leading to write the fluid acceleration along the path of a fluid element instead of along the particle trajectory. ${ }^{25}$ This difference should be inconsequential at low St. The Laplace force contribution is derived in the same way as the fluid-acceleration term, ${ }^{3,4}$ under the assumption that the magnetic field is uniform on the particle length scale. Equation (2) is valid for small particle radius and Reynolds number as well as small velocity gradients around the particle.

We now consider that the flow field $\mathbf{u}$ is a steady cellular flow field and write Eq. (2) in dimensionless form (denoted by the asterisks) using $k^{-1}$ as the lengthscale and the vortex velocity $U_{0}$ as the velocity scale,

$$
\begin{aligned}
\frac{d \mathbf{v}^{*}}{d t^{*}} & =\frac{1}{S t}\left(W \frac{\mathbf{g}}{|g|}+\mathbf{u}^{*}-\mathbf{v}^{*}+\frac{1}{6} P^{2} \nabla^{* 2} \mathbf{u}^{*}\right) \\
& +R\left(\frac{3}{2} \mathbf{u}^{*} \cdot \nabla \mathbf{u}^{*}+\frac{P^{2}}{20} \mathbf{u}^{*} \cdot \nabla \nabla^{* 2} \mathbf{u}^{*}\right)-\mathcal{L} \mathbf{j}^{*} \times \mathbf{B}^{*} \\
& +\frac{3}{\sqrt{2}} \sqrt{\frac{R}{S t}} \int_{0}^{t^{*}} \frac{-d \mathbf{v}^{*} / d \tau^{*}+\mathbf{v}^{*} \cdot \nabla \mathbf{u}^{*}+\frac{P^{2}}{6} \mathbf{v}^{*} \cdot \nabla \nabla^{* 2} \mathbf{u}^{*}}{\sqrt{\pi\left(t^{*}-\tau^{*}\right)}} d \tau^{*} .
\end{aligned}
$$

For solid spheres, $R=O(0.1)-O(1)$, and thus, for small $S t$, the drag-buoyancy term of order $O(1 / S t)$ [first term in the right-hand side of Eq. (3)] is dominant while the history term [third term in the right-hand side of Eq. (3)] is of higher order $O(1 / \sqrt{S t})$, and the Laplace term is of order $O(0.1 / S t)$ in our experiments, see Table IV. For most of the experiments, $P$ is always very small $(P \lesssim 0.16)$ and the Faxen corrections are negligible. For combination 1C (see Table IV), this is not the case as $P \approx 0.5$, and Faxen corrections may be discussed, as done in Sec. IV B. 
TABLE IV. Order of magnitude of the different terms of Eq. (3).

\begin{tabular}{lccccc}
\hline \hline St & & \multicolumn{3}{c}{ Added mass $^{\mathrm{c}}$} \\
& Acceleration $^{\mathrm{a}}$ & Drag-buoyancy $^{\mathrm{b}}$ & fluid acceleration & History $^{\mathrm{d}}$ & Laplace force $^{\mathrm{e}}$ \\
\hline 0.001 & $O(1)$ & $O(1000)$ & $O(0.1)-O(1)$ & $O(20)-O(70)$ & $O(10)-O(100)$ \\
0.01 & $O(1)$ & $O(100)$ & $O(0.1)-O(1)$ & $O(7)-O(20)$ & $O(1)-O(10)$ \\
0.1 & $O(1)$ & $O(10)$ & $O(0.1)-O(1)$ & $O(2)-O(7)$ & $O(0.1)-O(1)$ \\
1 & $O(1)$ & $O(1)$ & $O(0.1)-O(1)$ & $O(0.7)-O(2)$ & $O(0.01)-O(0.1)$ \\
\hline
\end{tabular}

${ }^{\mathrm{a}}$ Term in the left-hand side of Eq. (3).

${ }^{\mathrm{b}}$ First term in the right-hand side of Eq. (3).

${ }^{\mathrm{c}}$ Second term in the right-hand side of Eq. (3).

${ }^{\mathrm{d}}$ Fourth term in the right-hand side of Eq. (3).

${ }^{\mathrm{e}}$ Third term in the right-hand side of Eq. (3).

When $S t$ is very small (typically $S t \lesssim 0.01$ ), we recover the Stokes regime proposed by Stommel ${ }^{1}$ for which Eq. (3) reduces to

$$
\mathbf{v}^{*}=W \frac{\mathbf{g}}{|g|}+\mathbf{u}^{*}
$$

The velocity ratio $W$ is then the only control parameter. When the cellular flow consists of twodimensional Taylor-Green vortices given by the stream function (1), Eq. (4) yields

$$
\begin{aligned}
& \frac{d y_{1}^{*}}{d t^{*}}=\sin \left(y_{1}^{*}\right) \cos \left(y_{2}^{*}\right), \\
& \frac{d y_{2}^{*}}{d t^{*}}=-\sin \left(y_{2}^{*}\right) \cos \left(y_{1}^{*}\right)+W,
\end{aligned}
$$

where $\mathbf{y}$ is the position of the center of the particle and gravity is along direction 2 .

When examining the effect of a small but finite degree of inertia, Maxey ${ }^{2}$ neglected the history term in Eq. (3). Within this approximation, Eq. (3) becomes

$$
\frac{d \mathbf{v}^{*}}{d t^{*}}=\frac{1}{S t}\left(W \frac{\mathbf{g}}{|g|}+\mathbf{u}^{*}-\mathbf{v}^{*}+\frac{P^{2}}{6} \nabla^{2} \mathbf{u}^{*}\right)+R\left(\frac{3}{2} \mathbf{u}^{*} \cdot \nabla \mathbf{u}^{*}+\frac{P^{2}}{20} \mathbf{u}^{*} \cdot \nabla \nabla^{2} \mathbf{u}^{*}\right),
$$

which gives

$$
\begin{aligned}
& \frac{d^{2} y_{1}^{*}}{d t^{* 2}}=\frac{1}{S t}\left[\left(1-\frac{P^{2}}{3}\right) \sin \left(y_{1}^{*}\right) \cos \left(y_{2}^{*}\right)-\frac{d y_{1}^{*}}{d t^{*}}\right]+\frac{R}{2}\left(3-\frac{P^{2}}{5}\right) \sin \left(y_{1}^{*}\right) \cos \left(y_{1}^{*}\right), \\
& \frac{d^{2} y_{2}^{*}}{d t^{* 2}}=\frac{1}{S t}\left[-\left(1-\frac{P^{2}}{3}\right) \sin \left(y_{2}^{*}\right) \cos \left(y_{1}^{*}\right)-\frac{d y_{2}^{*}}{d t^{*}}+W\right]+\frac{R}{2}\left(3-\frac{P^{2}}{5}\right) \sin \left(y_{2}^{*}\right) \cos \left(y_{2}^{*}\right),
\end{aligned}
$$

for a Taylor-Green cellular flow. Note that $\mathrm{Maxey}^{2}$ also neglected the Faxen corrections and thus considered the above equations with $P=0$. Again, for $R=O(0.1)$ to $O(1)$ and for $S t \ll 1$, the drag-buoyancy term (first term in the right-hand side of the equations) is largely dominant in Eqs. (6) or (7). Neglecting the history term may be valid for this range of small $S t$ but is disputable when $S t \gtrsim 0.1$ as it can be of the same order of magnitude as the drag-buoyancy term. When $S t \sim$ 1, all the different terms of Eq. (3) become of the same order of magnitude as shown in Table IV. However, the validity of the Boussinesq-Basset-Oseen equation (2) needs to be assessed, since the Reynolds number may not be small.

The above equations proposed by Stommel ${ }^{1}$ and Maxey $^{2}$ can be easily solved numerically, both for a Taylor-Green cellular flow [Eqs. (5) or (7)], and for a cellular flow $\mathbf{u}$ inferred from the interpolated PIV measurements [Eqs. (4) or (6)]. Starting from the experimentally-known initial position of the particle [and with the additional knowledge of its velocity for solving equations (6) and (7)], its subsequent positions are calculated using a Runge-Kutta 4th/5th order scheme (ode45 in Matlab ${ }^{\mathrm{TM}}$ ) and stored at chosen time intervals. The numerically-obtained trajectory of the particle can then be compared to the experimental observations. The Laplace force can be easily added to the Stommel or Maxey equations and its influence examined. Accounting for the 
history force is more difficult and necessitates a different numerical scheme since it involves the calculation of integrals with singular, but integrable, integrands. We have used the recent second order scheme proposed by Daitche. ${ }^{26}$

\section{PARTICLE MOTIONS}

\section{A. Stokes regime $S t \lesssim 0.01$ and $R e_{k}<1$}

First, we present the experimental trajectories obtained for very small $S t$ and for varying $W$ (combinations $1 \mathrm{~A}, 1 \mathrm{~B}$, and $1 \mathrm{C}$ in Table $\mathrm{III}$ ) and compare them to those obtained from solving equations (5) using the corresponding $W$ and (7) using the corresponding $W, R, P$, and $S t$ in Table III.

When $W$ is quite large ( $W=21.646$ for combination 1C), the particles settle through the vortex array and the trajectories are straight vertical lines as shown for a typical trajectory in Figure 5

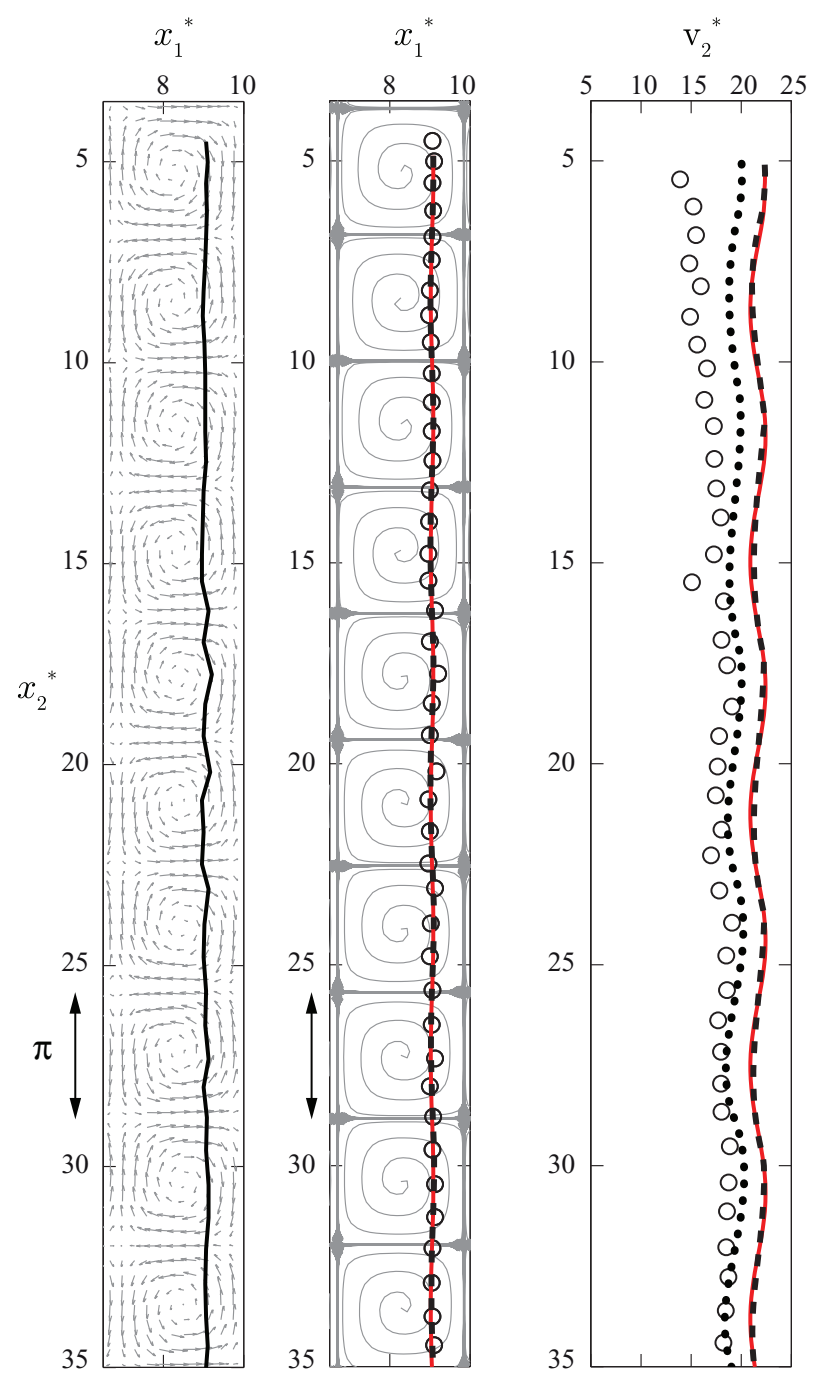

FIG. 5. (Left panel) a typical experimental trajectory (black solid line) for combination 1C in the velocity-vector map of the vortical flow. (Middle panel) comparison of the experimental trajectory ( 0 ) with the simulations in the Taylor-Green flow-lines [dashed line (black) solving equation (5) and solid line (red) solving equation (7) using the dimensionless numbers given in Table III for combination 1C]. (Right panel) comparison of the experimental velocity (along gravity) and the simulations [dashed line (black) solving equation (5), solid line (red) solving equation (7) using the dimensionless numbers given in Table III for combination 1C, and dotted line (black) solving equation (5) using $\left.W_{\exp }=19.341\right]$. 
(left and middle panels). The vertical velocity (right panel) exhibits small oscillations due to the small modulation caused by the vortical flow. The numerical trajectory (dashed line) obtained with Eq. (5) is in excellent agreement with the experimental trajectory (middle panel). The numerical prediction for the vertical velocity presents similar oscillations as those observed experimentally but the velocity magnitude is slightly overestimated (right panel). Better agreement is obtained when the velocity ratio is taken as $W_{\exp }=19.341$ (black dotted line) instead of $W=21.646$ (black dashed line). This may be due to the fact that the particle Reynolds number is slightly larger than one for this combination $1 \mathrm{C}\left(R e_{a}=3.472\right)$ and thus that the experimental drag is slightly larger than the Stokes drag (see Figure 4). This slightly larger drag is likely due to wall effect for this large particle $(a=0.3161 \mathrm{~cm})$ settling in the vicinity of the back wall of the tank.

When $W$ is larger than unity but not so large ( $W=3.21$ for combination $1 \mathrm{~B})$, the particles released at different locations at the top of the vortex arrays are all settling through the down-flow region of successive vortices and display zigzagging motions [see Figure 6 (left and middle panels)]. The motion of the particles is very reproducible when released at the same location as shown in the accompanying movie (see Figure 6) (Multimedia view). The vertical velocities also present periodic oscillations due to the modulations caused by the periodic cellular flow [see Figure 6 (right panel)]. Interestingly, when particles are released at the border between two neighboring vortices,
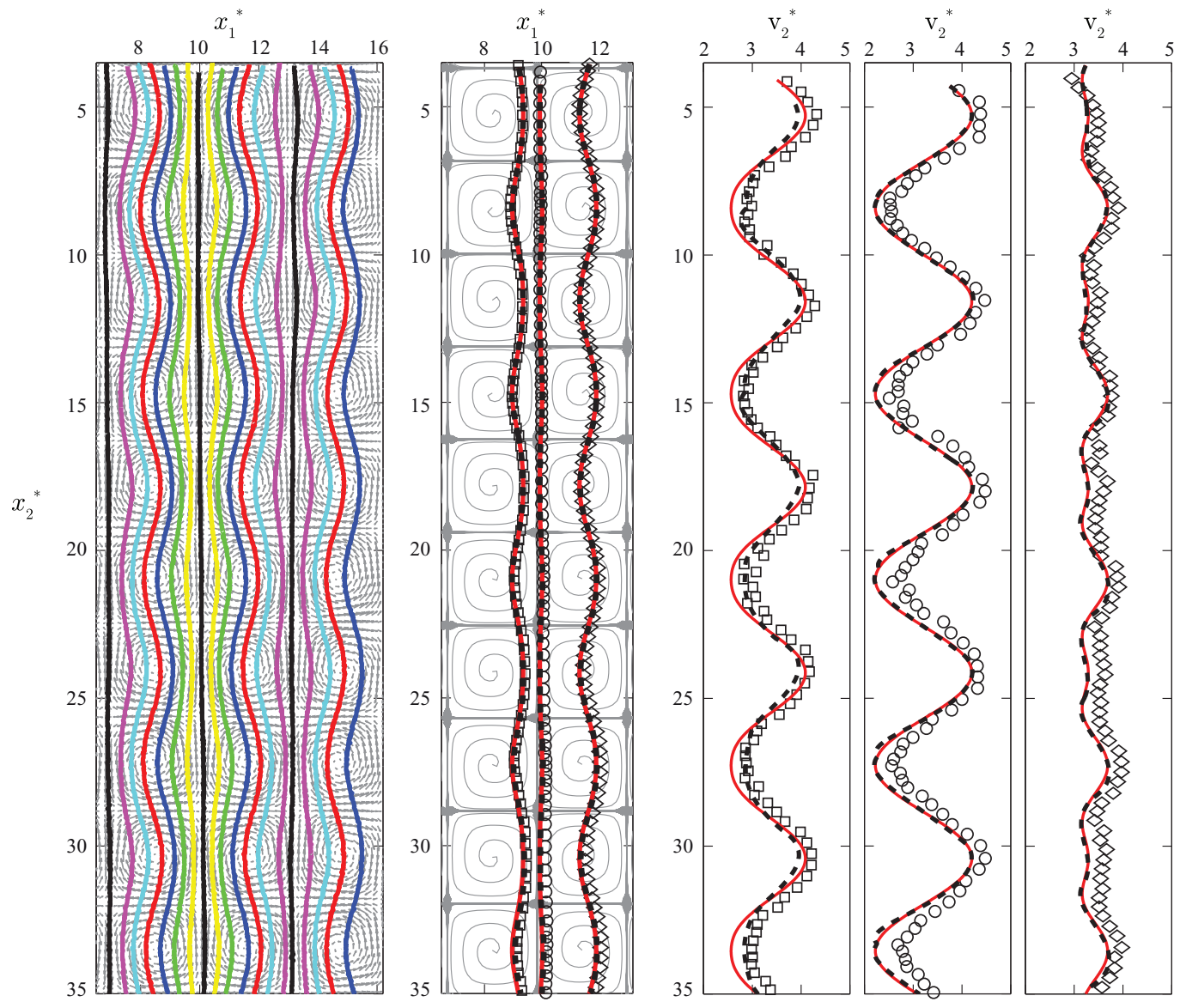

FIG. 6. (Left panel) experimental trajectories (solid lines having different color) for combination 1B in the velocity-vector map of the vortical flow - see accompanying movie. (Middle panel) comparison of 3 typical experimental trajectories ( $\square$, o, $\diamond$ ) with the simulations in the Taylor-Green flow-lines [dashed line (black) solving equation (5) and solid line (red) solving equation (7) using the dimensionless numbers given in Table III for combination 1B]. (Right panel) comparison of the experimental velocities (along gravity) corresponding to the 3 typical experimental trajectories shown in the middle panel ( $\square$, $\circ, \diamond$ ) and the simulations [dashed line (black) solving equation (5), solid line (red) solving equation (7) using the dimensionless numbers given in Table III for combination 1B]. (Mutimedia view) [URL: http://dx.doi.org/10.1063/1.4895736.1]. 

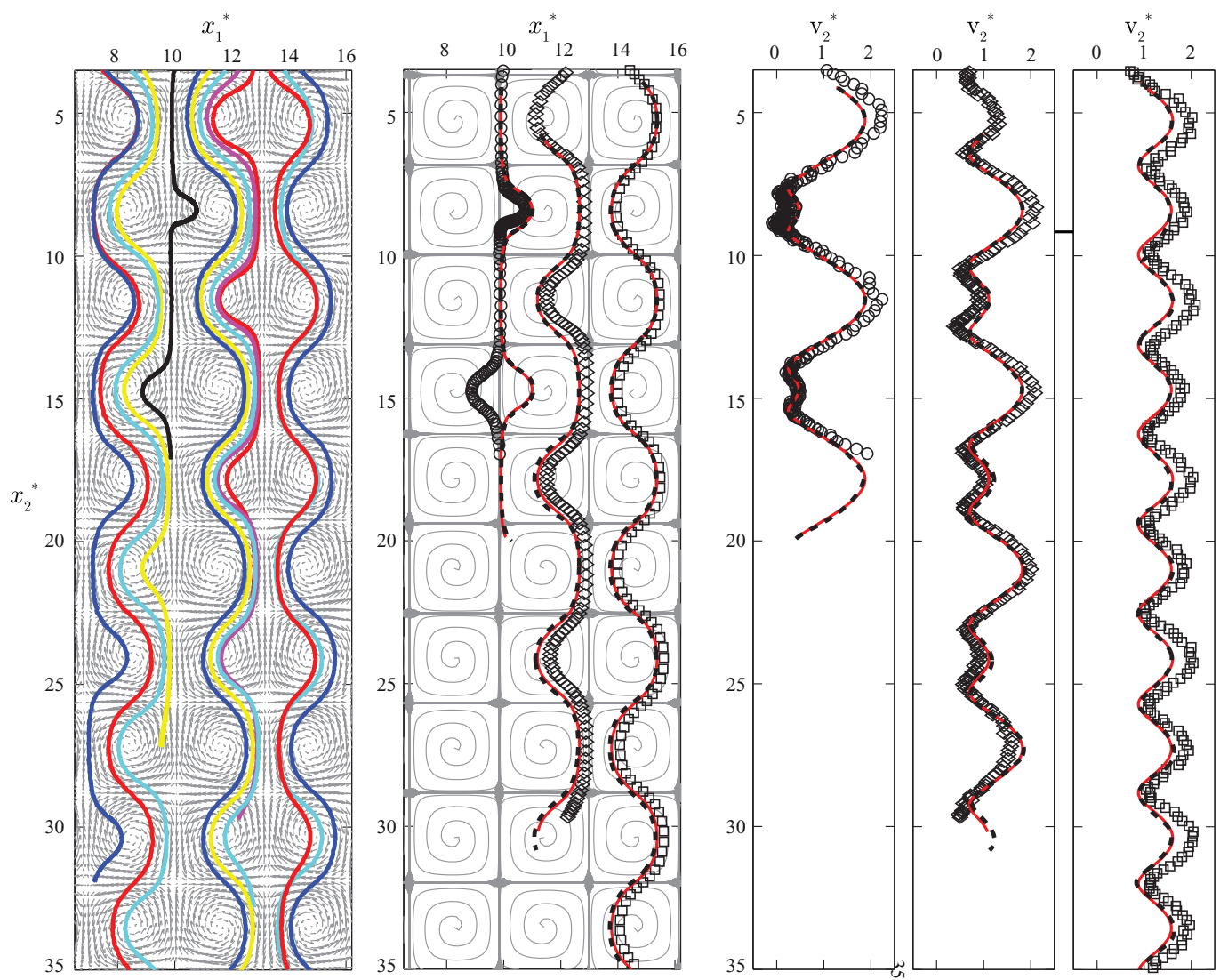

FIG. 7. Same as Figure 6 but for combination 1A.

the trajectory is nearly a straight line (black solid lines in left panel and $\circ$ in middle panel) but the corresponding velocity ( $\mathrm{o}$ in right panel) exhibits strong oscillation as the particle is going through the successive stagnation points. Good agreement is obtained between the experimental observations for 3 typical trajectories and the simulations using Eq. (5) with $W=3.21$ (dashed lines in middle and right panels).

When $W$ is below unity ( $W=0.878$ for combination $1 \mathrm{~A}$ ), the particles are still all settling out and both the trajectories and vertical velocities show even more pronounced oscillations as can be seen in Figure 7. When they are released at the border between two neighboring vortices, they experience a strong slowdown and deviate from their straight path by making small excursions as if to avoid the successive upward-flow regions (black solid lines in left panel and $\circ$ in middle panel). The numerical simulations using Eq. (5) with $W=0.878$ (dashed lines in middle and right panels) show good agreement with the experimental observations for 3 typical trajectories. The only detected discrepancy occurs for the trajectory discussed above ( $\circ$ in middle panel) for which the numerical simulation predicts small periodic excursions always on the same side, while it can be on one side or the other for the experiments due to a great sensitivity to the local velocity field, in particular at the stagnation (or saddle) points. This is reminiscent of Lagrangian chaos observed for neutrally buoyant particles $(W=0) .{ }^{20}$ The experimental variations of the velocity is nonetheless well captured by the simulations in this later case.

The simulations using Eq. (7) with the corresponding dimensionless parameters of Table III provide similar results to those using Eq. (5) [see solid (red) lines in Figures 5-7]. Using $R$ and $P=$ 0 does not change the predictions. Similarly, adding the Laplace and history forces does not affect the results. The simulations are also insensitive to the choice of initial velocity. This confirms that the drag-buoyancy term is dominant and that, in this range of very low $S t$ and small $R_{e_{k}}$ (and in 
addition small $R e_{a}$ ), the particle velocity can be described by the sum of the fluid velocity and its Stokes velocity such as given by Eq. (4). Inertia is thus negligible and the regime is that of Stokes.

\section{B. Weak inertia $0.01 \lesssim S t<0.1$ and $R e_{k} \approx 6-8$}

We now turn to experimental trajectories obtained for larger $S t$ and $\operatorname{Re}_{k}$ (combinations $2 \mathrm{~B}$ and 3B in Table III) and compare them to those obtained from solving equations (4) with the corresponding $W$ and (6) using the corresponding $W, R, P$, and $S t$ in Table III.

When $W$ is larger than unity ( $W=4.133$ for combination $2 \mathrm{~B}$ ), the trajectories tend to drift toward the border lines between the cells and have oscillations with diminishing amplitude as the particles are settling through the successive vortices [see Figure 8 (left panel)]. The trajectory drift and decrease in amplitude is not reproduced by the simulations using Eq. (5) with $W=4.133$. The drift can be captured by using the interpolated velocity field given by the PIV measurements instead of the Taylor-Green flow-field (Figure 8 middle panel) as, for large $\operatorname{Re}_{k}(=8.017)$, the cellular flow produced by electroconvection deviates from a Taylor-Green flow, see Figures 2(a) and 2(c). Excellent agreement including realistic decrease in amplitude is obtained when the velocity ratio is taken as $W_{\exp }=2.474$ or equivalently $W_{\mathrm{SN}}=2.63$ [black dotted line in Figure 8 (middle and right panels)]. The trajectories using the Schiller-Naumann drag lie between those using the Stokes (black dashed line) and Oseen (black dashed-dotted line) drags [ $W_{\text {Stokes }}=W=4.133$ and $\left.W_{\text {Oseen }}=1.398\right]$.
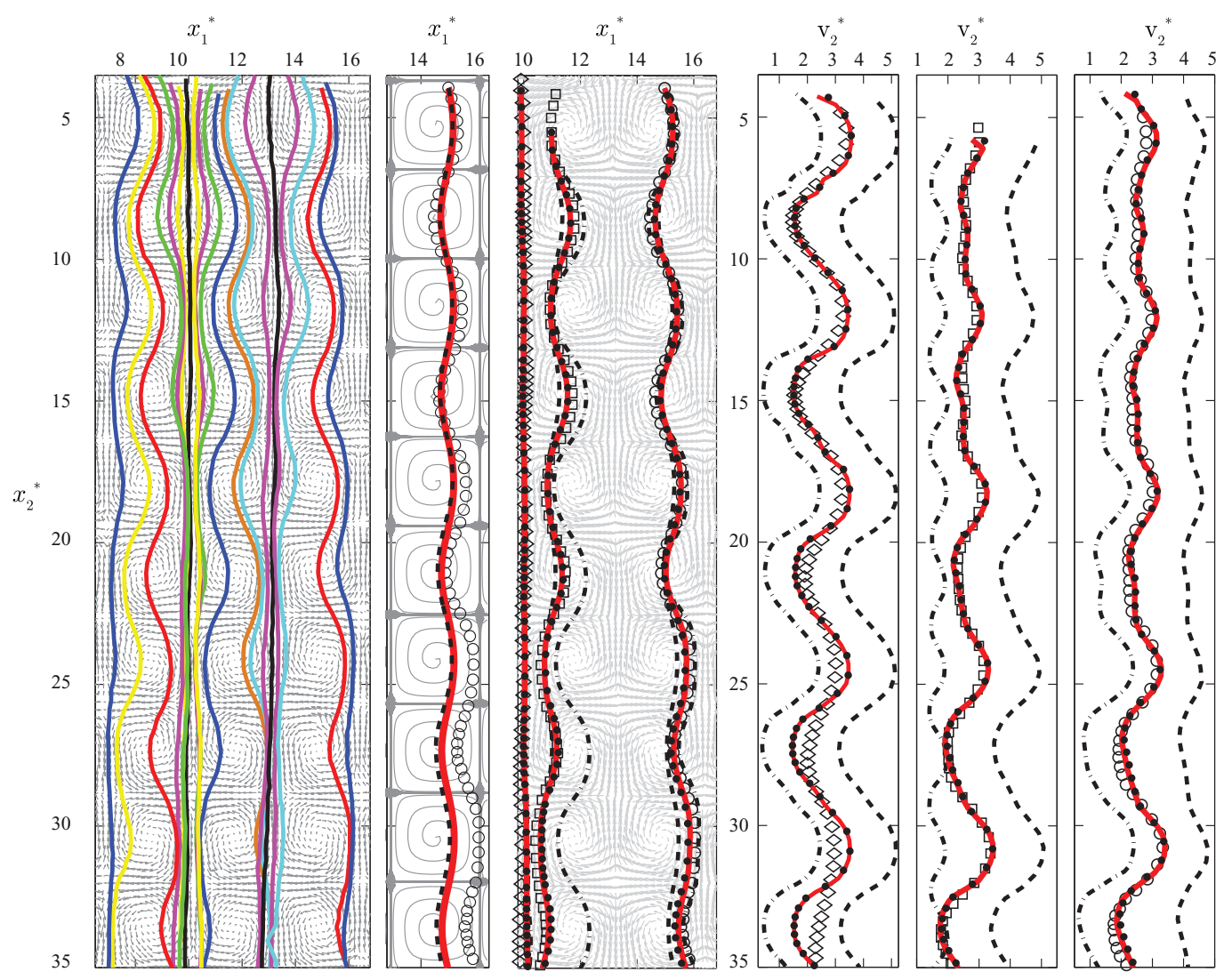

FIG. 8. Same as Figure 6 but for combination 2B. The middle panel comprises a comparison of a typical experimental trajectory (o) with the simulations in the Taylor-Green flow-lines [dashed line (black) solving equation (5) and solid line (red) solving equation (7) using the corresponding $W, R, P$, and $S t$ in Table III for combination 2B] and a comparison of 3 typical experimental trajectories $(\square, \circ, \diamond)$ with the simulations using the interpolated PIV flow-field [dashed line (black) solving equation (4) with $W=W_{\text {Stokes }}=4.133$, dotted line (black) with $W_{\text {exp }}=2.474$, dashed-dotted line (black) with $W_{\text {Oseen }}=1.398$, and solid line (red) solving equation (6) using the corresponding $W_{\exp }, R, P$, and $S t_{\exp }$ in Table III for combination $2 \mathrm{~B}$ ]. The right panel also includes comparison with the same simulations using the interpolated PIV flow-field. 

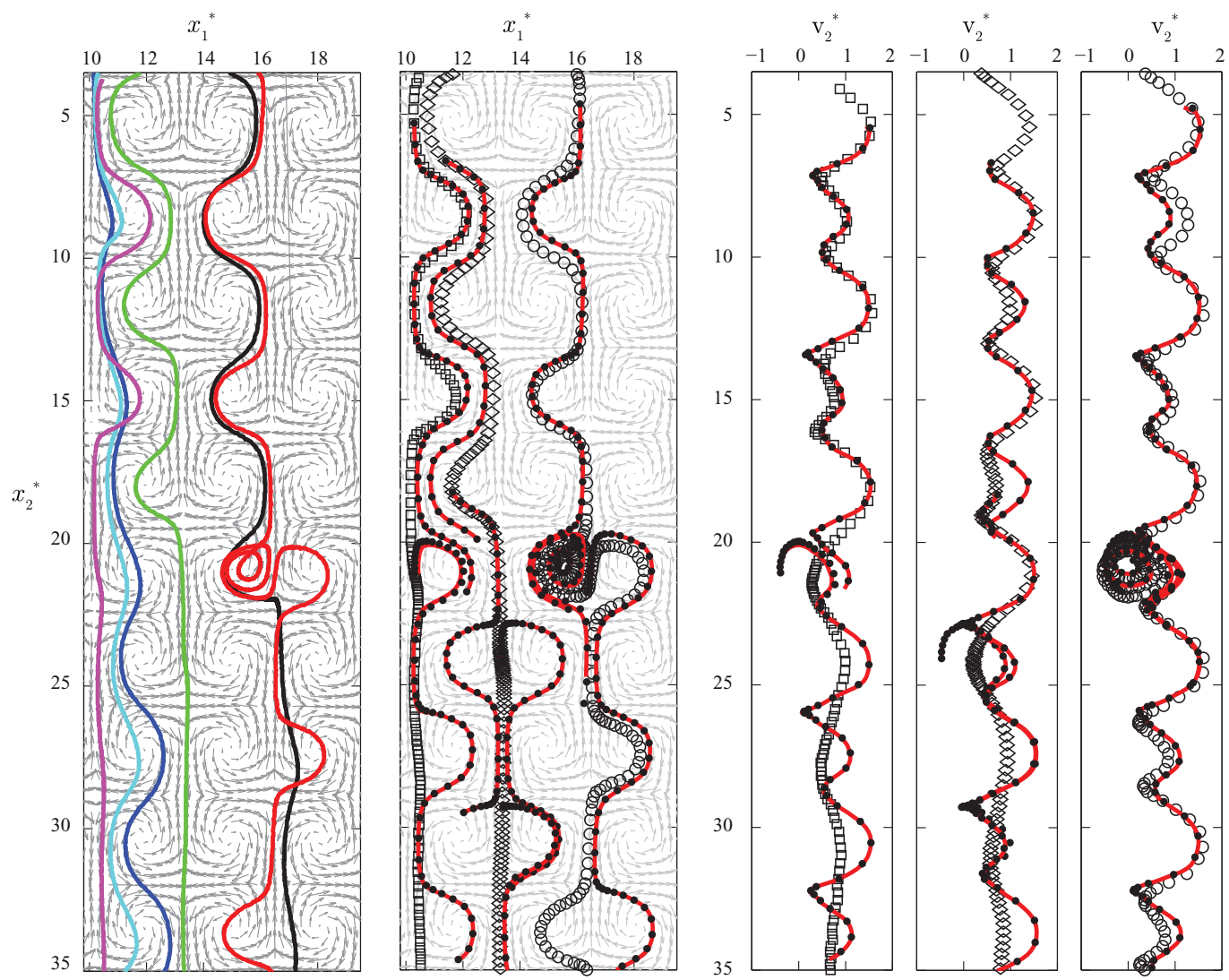

FIG. 9. Same as Figure 8 but for combination 3B - see accompanying movie. The comparison only concerns a single typical trajectory and the comparison is only shown with the simulations using the interpolated PIV flow-field [dotted line (black) solving equation (4) using $W_{\exp }=0.530$ and solid line (red) solving equation (6) using the corresponding $W_{\exp }, R, P$, and $S t_{\exp }$ in Table III for combination 3B]. (Multimedia view) [URL: http://dx.doi.org/10.1063/1.4895736.2]

Clearly, since $R e_{a}=5.221$ and is larger than unity, the drag deviates from the linear Stokes drag. It is larger than the Stokes drag and smaller than the Oseen drag (see Figure 4).

When $W$ is much smaller than unity ( $W=0.667$ for combination $3 \mathrm{~B}$ ), the particles are still settling out but can become trapped in a cell and make a few loops before being released and falling out again as shown for a typical trajectory in Figure 9. This behavior can be well captured by the simulations using Eq. (4) with the interpolated velocity field given by the PIV measurements and the experimental velocity ratio $W_{\exp }=0.530$ or the Schiller-Naumann velocity ratio $W_{\mathrm{SN}}=0.567$. Note that a numerical relative tolerance of $10^{-5}$ was used in all the simulations and was found to be critical to reproduce the trapping of the particle in this latter case. Note also that, because of the great sensitivity to initial position as previously observed for combination $1 \mathrm{~A}$, the trajectories can be well captured only for short duration (typically during the sampling of 2-3 vortices). Trapping is more difficult to reproduce as the particle probes a saddle point at each loop.

It is interesting at that point to discuss further the trapping dynamics shown in Figure 9. The existence of "region of retention" for which closed particle trajectories exist in the range $0 \leqslant W \leqslant 1$ was discussed by Stommel ${ }^{1}$ (see his Figure 4). This was revisited by Maxey ${ }^{2}$ who showed that particle inertia suppressed these regions as no permanent suspension occurred (see, e.g., his Figure 7). In the present experimental conditions, no permanent trapping was observed. We numerically investigated the dynamics of individual particles during their settling through the experimental vortical flow of Figure 9 . The two top cells were uniformly seeded and the particles followed up to $40 / k U_{0}$. As shown in the movie of the supplementary material, ${ }^{27}$ the particles are seen to escape the trapping zones and to accumulate along asymptotic trajectories. In the present experimental conditions, the lack of 
resuspension is not due to inertia but to the real vortical flow which differs from a Taylor-Green flow and leads to a drift of the trajectories as mentioned earlier in discussing Figure 8.

To conclude this section, the simulations using Eq. (6) provide again similar results to those using Eq. (4) [see solid (red) lines in Figures 8 and 9]. Moreover, the predictions are similar using $R=P=\mathcal{L}=0$ and are insensitive to the choice of initial velocities. The regime is no longer that of Stokes but, since the drag-buoyancy term is largely dominant, the particle velocity can be simply described by the sum of the fluid velocity and the finite- $R e_{a}$ particle settling velocity.

\section{Influence of the history force}

Several numerical studies have aimed at characterizing the influence of the different terms in the full Boussinesq-Basset-Oseen model (3), and in particular that of the history force. ${ }^{9,11,13}$ This force is found to be relevant in many cases involving significant particle acceleration, in particular in instationary flows. ${ }^{28-31}$ A general result is the weakening of attracting regions of the flow, reducing entrapment of particles lighter than the fluid; this was observed experimentally for bubbles. ${ }^{32}$ In a recent numerical work, Olivieri and co-workers ${ }^{31}$ showed in homogeneous and isotropic turbulence that the history force was responsible for up to $10 \%$ of particle acceleration, in particular during strong (and rare) events. This is in fact crucial, as this force plays a role essentially when the particle is submitted to significant accelerations. Candelier and co-workers ${ }^{28}$ addressed this issue experimentally by placing particles inside a solid-body vortex. They measured up to a $10 \%$ overestimation of the ejection rate when the history force was not accounted for. The particularity of this study is that the particles were under constant acceleration due to the solid-body rotation.

In the original work of Maxey, ${ }^{2}$ the history force was considered as negligible. We have nonetheless examined the influence of this force in the present experimental conditions, and in particular for the largest Stokes and Reynolds numbers where its influence should be the most significant as explained in Sec. III when discussing Table IV. This influence is probed in Figure 10 for combination $2 \mathrm{~B}$ and more precisely for the trajectory positioned on the right in the middle panel of Figure 8. The time evolution of the particle position shows clearly that the Schiller-Naumann drag is sufficient to recover properly the trajectories. In particular, the amplitude and phase of the oscillations is nicely captured (see bottom graph). Conversely, the simple Stommel model (4) with
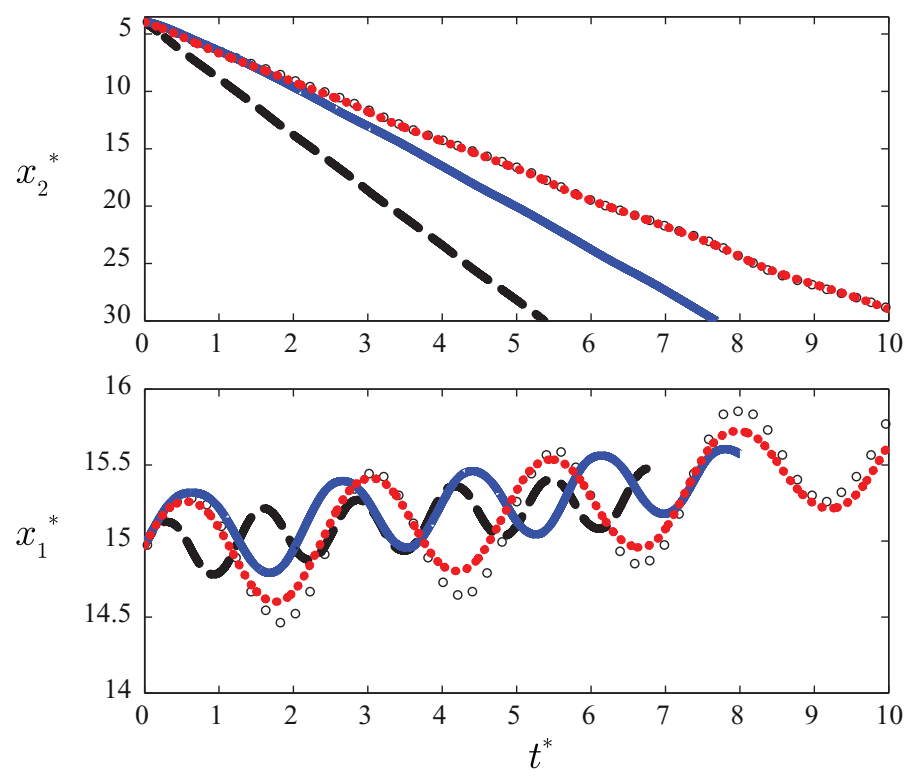

FIG. 10. Time evolution of vertical (top) and horizontal (bottom) particle coordinates for the trajectory positioned on the right in the middle panel of Figure 8. Comparison between Stommel (black dashed line), Boussinesq-Basset-Oseen (blue line) models, and Stommel corrected with the Schiller-Naumann drag (red dotted line) with the experiments (o). 
a Stokes drag and the full Boussinesq-Basset-Oseen model (3) derived in the Stokes regime both overestimate the settling velocity. Thus, they are not able to capture the oscillations of the particle. It is interesting to note that the full Boussinesq-Basset-Oseen model is only able to reproduce satisfactorily the first oscillation.

Clearly, the order of magnitude of the history force is smaller than that of the nonlinear dragbuoyancy term. In addition, the history force is meant to capture well short time accelerations which in fact are not important in the present experimental conditions. The comparison with the experiments shows that the major ingredient to be included in the model is the nonlinear Schiller-Naumann drag, and leads to questioning the validity of the Boussinesq-Basset-Oseen model (3).

\section{CONCLUSION}

In this work, we have presented a jointed experimental and numerical study of the settling of small solid spheres in a cellular flow field at low Stokes number. At very low Stokes number $(<0.01)$, we have identified a Stokes regime for which the velocity of the particle is simply the sum of its Stokes velocity and the local fluid velocity given by the cellular flow. The trajectories are discussed in term of a single dimensionless parameter, the ratio of the Stokes velocity to the vortex velocity. At larger Stokes number (but still $<0.1$ ), the particle velocity is still described by the sum of the fluid velocity and the particle settling velocity. However, as the particle Reynolds number can be larger than unity, the drag is no longer the Stokes drag but becomes nonlinear and can be well reproduced by correlations such as that of Schiller-Naumann. Again, the particle trajectories can be analyzed in terms of the same dimensionless number, the velocity ratio. Overall, when this velocity ratio is much larger than unity, the particle trajectories are straight lines and the particles experience only small modulations coming from the vortical flow. When this ratio is much smaller than unity, the particles are settling out but can become momentarily trapped in a cell. In between, the particle trajectories present more or less marked oscillations. In the regimes of Stokes number that we have explored in this paper, added mass, Laplace, and history forces are negligible, and drag and buoyancy prevail.

\section{ACKNOWLEDGMENTS}

This work was undertaken under the auspices of ANR project "Collective Dynamics of Settling Particles In Turbulence" (ANR-12-BS09-0017-01), CNRS PICS project "Experimental and numerical study of finite-inertia effects in sedimenting suspensions" (CNRS-PICS05848), "Laboratoire d'Excellence Mécanique et Complexité" (ANR-11-LABX-0092), and "Initiative d'Excellence" A*MIDEX (ANR-11-IDEX-0001-02). It benefited from the stay of A. Martin in our laboratory and from the help of F. Candelier in implementing the history force. It also benefited from discussions with E. Climent, A. Daitche, O. du Roure, A. Lindner, M. R. Maxey, A. Pocheau, and A. Soldati.

${ }^{1}$ H. Stommel, "Trajectories of small bodies sinking slowly through convection cells," J. Mar. Res. 8, 24-29 (1949).

${ }^{2}$ M. R. Maxey, "The motion of small spherical particles in a cellular flow field," Phys. Fluids 30, 1915-1928 (1987).

${ }^{3}$ R. Gatignol, "The Faxén formulae for a rigid particle in an unsteady non-uniform stokes flow," J. Méca. Théo. Appli. 2, 143-160 (1983).

${ }^{4}$ M. R. Maxey and J. J. Riley, "Equation of motion for a small rigid sphere in a non-uniform flow," Phys. Fluids 26, 883-889 (1983).

${ }^{5}$ F. Toschi and E. Bodenschatz, "Lagrangian properties of particles in turbulence," Annu. Rev. Fluid Mech. 41, 375-404 (2009).

${ }^{6}$ S. Balachandar and J. K. Eaton, “Turbulent dispersed multiphase flow," Annu. Rev. Fluid Mech. 42, 111-133 (2010).

${ }^{7}$ L.-P. Wang and M. R. Maxey, "Settling velocity and concentration distribution of heavy particles in homogeneous isotropic turbulence," J. Fluid Mech. 256, 27-68 (1993).

${ }^{8}$ M. Maxey and S. Corrsin, "Gravitational settling of aerosol particles in randomly oriented cellular flow fields," J. Atmos. Sci. 43, 1112-1134 (1986)

${ }^{9}$ S. Elghobashi and G. Truesdell, "Direct simulation of particle dispersion in a decaying isotropic turbulence," J. Fluid Mech. 242, 655-700 (1992).

${ }^{10} \mathrm{C}$. Yang and U. Lei, "The role of the turbulent scales in the settling velocity of heavy particles in homogeneous isotropic turbulence," J. Fluid Mech. 371, 179-205 (1998).

${ }^{11} \mathrm{~V}$. Armenio and V. Fiorotto, "The importance of the forces acting on particles in turbulent flows," Phys. Fluids 13, 2437-2440 (2001). 
${ }^{12}$ T. Bosse, L. Kleiser, and E. Meiburg, "Small particles in homogeneous turbulence: Settling velocity enhancement by two-way coupling," Phys. Fluids 18, 027102 (2006).

${ }^{13}$ Y. Ling, M. Parmar, and S. Balachandar, "A scaling analysis of added-mass and history forces and their coupling in dispersed multiphase flows," Int. J. Multiphas. Flow 57, 102-114 (2013).

${ }^{14}$ J. R. Fessler, J. D. Kulick, and J. K. Eaton, "Preferential concentration of heavy particles in a turbulent channel flow," Phys. Fluids 6, 3742-3749 (1994).

${ }^{15}$ R. Monchaux, M. Bourgoin, and A. Cartellier, "Analyzing preferential concentration and clustering of inertial particles in turbulence," Int. J. Multiphas. Flow 40, 1-18 (2012).

${ }^{16}$ Y. Tagawa, J. M. Mercado, V. N. Prakash, E. Calzavarini, C. Sun, and D. Lohse, “Three-dimensional lagrangian Voronoï analysis for clustering of particles and bubbles in turbulence," J. Fluid Mech. 693, 201-215 (2012).

${ }^{17}$ G. Ruetsch and E. Meiburg, "On the motion of small spherical bubbles in two-dimensional vortical flows," Phys. Fluids A 5, 2326-2341 (1993).

${ }^{18}$ C. Marchioli, M. Fantoni, and A. Soldati, "Influence of added mass on anomalous high rise velocity of light particles in cellular flow field: A note on the paper by Maxey (1987)," Phys. Fluids 19, 098101 (2007).

${ }^{19}$ P. Tabeling, B. Perrin, and S. Fauve, "Instability of a linear array of forced vortices," Europhys. Lett. 3, 459 (1987).

${ }^{20}$ N. T. Ouellette, P. J. J. O’Malley, and J. P. Gollub, “Transport of finite-sized particles in chaotic flow," Phys. Rev. Lett. 101, 174504 (2008).

${ }^{21}$ E. Wandersman, N. Quennouz, M. Fermigier, A. Lindner, and O. Du Roure, "Buckled in translation," Soft Matter 6, 5715-5719 (2010).

${ }^{22}$ P. Meunier and T. Leweke, "Analysis and treatment of errors due to high velocity gradients in Particle Image Velocimetry," Exp Fluids 35, 408-421 (2003).

${ }^{23}$ J. Paret, D. Marteau, O. Paireau, and P. Tabeling, "Are flows electromagnetically forced in thin stratified layers two dimensional?" Phys. Fluids 9, 3102-3104 (1997).

${ }^{24}$ D. H. Kelley and N. T. Ouellette, "Onset of three-dimensionality in electromagnetically driven thin-layer flows," Phys. Fluids 23, 045103 (2011).

${ }^{25}$ T. Auton, J. Hunt, and M. Prud'Homme, "The force exerted on a body in inviscid unsteady non-uniform rotational flow," J. Fluid Mech. 197, 241-257 (1988).

${ }^{26}$ A. Daitche, "Advection of inertial particles in the presence of the history force: Higher order numerical schemes," J. Comput. Phys. 254, 93-106 (2013).

${ }^{27}$ See supplementary material at http://dx.doi.org/10.1063/1.4895736 for movie for simulation of dynamics of individual particles during their settling through the experimental vortical flow.

${ }^{28}$ F. Candelier, J. Angilella, and M. Souhar, "On the effect of the Boussinesq-Basset force on the radial migration of a stokes particle in a vortex," Phys. Fluids 16, 1765-1776 (2004).

${ }^{29}$ A. Daitche and T. Tél, "Memory effects are relevant for chaotic advection of inertial particles," Phys. Rev. Lett. 107, 244501 (2011).

${ }^{30} \mathrm{~K}$. Guseva, U. Feudel, and T. Tél, "Influence of the history force on inertial particle advection: Gravitational effects and horizontal diffusion,” Phys. Rev. E 88, 042909 (2013).

${ }^{31}$ S. Olivieri, F. Picano, G. Sardina, D. Iudicone, and L. Brandt, "The effect of the Basset history force on particle clustering in homogeneous and isotropic turbulence," Phys. Fluids 26, 041704 (2014).

${ }^{32}$ R. Toegel, S. Luther, and D. Lohse, "Viscosity destabilizes sonoluminescing bubbles," Phys. Rev. Lett. 96, 114301 (2006). 